\title{
A Osteoporose e a Mulher Envelhecida: fatores de risco
}

Osteoporosis and Aged Women: risk factors

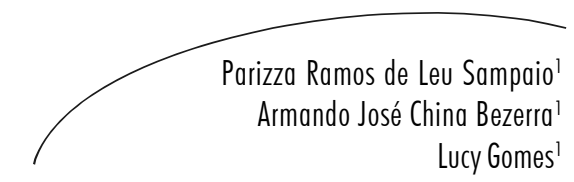

Resumo

A osteoporose é uma doença silenciosa, o que implica reconhecer os fatores de risco inerentes a cada mulher para se determinar estratégias de melhoria da qualidade de vida. Objetivo: verificar a chance de as mulheres terem fatores de risco para osteoporose, através do questionário proposto pelo estudo do European Vertebral Osteoporosis Study Group (EVOS) e avaliar o desempenho nas atividades da vida diária em relação às ações mais executadas no dia a dia pelo questionário Atividades Instrumentais da Vida Diária (AIVD). O exame da densitometria óssea foi realizado para diagnosticar a osteoporose. Metodologia: aplicaram-se a ficha com dados sociodemográficos e o questionário EVOS no primeiro tempo e após o questionário de AIVD e o exame de densitometria óssea nas mulheres na pósmenopausa, com idade $=60$ anos. Resultados: foram analisadas 58 mulheres, com idade média de 67,3 anos. O índice de massa corporal (IMC), um dos fatores de risco, foi considerado fator preditor para ausência de osteoporose, pois as mulheres deste estudo apresentavam sobrepeso. Dezesseis (28\%) apresentavam osteoporose, com escores médios significativamente menores que aquelas que não têm osteoporose, para os itens: consegue fazer compras $(p=0,006)$, consegue fazer trabalhos manuais $(p=0,018)$ e cuidar das finanças $(p=0,014)$. Conclusão: a variável IMC na regressão logística teve relevância no questionário EVOS. Na análise do questionário de AIVD, as mulheres com osteoporose apresentam significância em relação às que não tinham osteoporose, para os itens: consegue fazer compras, consegue fazer trabalhos manuais e cuidar das finanças.

\section{Abstract}

Osteoporosis is a silent disease which involves the recognition of risk factors inherent to every woman in the determination of strategies to improve the quality of life. Objective: to analyze the chance of women having risk factors for osteoporosis using the questionnaire proposed by the EVOS study and evaluate the performance

Programa de pós-graduação Stricto Sensu em Gerontologia, Universidade Católica de Brasília. Brasília, DF, Brasil

Palavras-chave: Seviços de saúde a mulher. Saúde da terceira idade. Qualidade de vida. Idoso.

Osteoporose.

Densitometria. 
in activities of daily living in relation to actions performed on most days by the IADLs questionnaire. The examination of bone densitometry was performed to diagnose osteoporosis. Methodology: we applied the plug with socio-demographic data and questionnaire EVOS the first time and after the IADLs questionnaire and examination of bone densitometry in postmenopausal women, aged $=60$ years. Results: we analyzed 58 women with a mean age of 67.3 years. The BMI, a risk factor, was considered as a predictor for the absence of osteoporosis, since women in this study were overweight. Sixteen (28\%) had osteoporosis, with mean scores significantly lower than those who do not have osteoporosis of items: get shopping $(p=0.006)$, manages to do manual labor $(p=0.018)$ and taking care of finances $(p=0.014)$. Conclusion: the BMI variable in the logistic EVOs had relevance in the questionnaire. In the questionnaire analysis of IADLs, women with osteoporosis showed significance for those without osteoporosis for the items: go shopping, can make crafts and take care of finances.
Key words: Women's Health Services. Health of the Elderly. Quality of Life. Aged. Osteporosis. Densitometry.

\section{INTRODUÇÃO}

O Brasil caminha velozmente rumo a perfil demográfico cada vez mais envelhecido, fenômeno que implicará adequações nas políticas sociais, particularmente naquelas destinadas a atender as crescentes demandas dessa parcela da população nas áreas da saúde, previdência e assistência social. ${ }^{1}$

Climatério é definido como a fase da vida biológica da mulher que marca a transição entre o período reprodutivo e o não-reprodutivo, isto é, da menarca para a senilidade. ${ }^{2}$ A menopausa é evento singular, correspondendo ao último período menstrual, tendo seu diagnóstico firmado após um ano de amenorreia. ${ }^{3}$ Mundialmente, até o ano 2030, haverá um bilhão e 200 milhões mulheres vivendo a menopausa. ${ }^{4}$

$\mathrm{Na}$ última década, verificou-se que o estrogênio, hormônio reprodutivo feminino, afeta enorme quantidade de funções, incluindose o metabolismo ósseo e mineral. ${ }^{5}$ Osteoporose é enfermidade crônica e multifatorial, relacionada ao envelhecimento, caracterizada por baixa massa óssea e deterioração da microarquitetura do tecido ósseo, conduzindo ao aumento da fragilidade dos ossos e, consequentemente, à elevação do risco de fraturas, o que a torna problema de Saúde Pública. ${ }^{2,6}$

A osteopenia é caracterizada somente por perda da massa óssea, sem intercorrência de fraturas. ${ }^{7}$ Apesar de a perda óssea após a menopausa ser maior no osso trabecular, como consequência da queda brusca da concentração de estrógenos, outros fatores afetam de modo progressivo e mais lento essa perda. tanto no osso cortical quanto no trabecular. ${ }^{8}$

As osteoporoses pós-menopáusica e a senil são formas primárias de osteoporose consideradas involucionais. $\mathrm{Na}$ menopausa, a cada ano é perdido de $1 \%$ a $3 \%$ do osso cortical e acima de $5 \%$ do osso trabecular. O adelgaçamento das trabéculas pode levar à incapacidade parcial ou permanente, diminuindo a independência física e social dos pacientes acometidos por osteoporose. ${ }^{9}$ É doença tratável e capaz de ser prevenida, embora a fratura possa levar à incapacidade permanente. Portanto, a prevenção e a detecção precoce da osteoporose devem ser objetivos da abordagem clínica do climatério. ${ }^{10}$

As fraturas podem ser vertebrais e nãovertebrais (principalmente no fêmur, punho e costelas). As mais incapacitantes são as do fêmur, que causam a morte em quase $25 \%$ dos pacientes no primeiro ano pós-fratura. ${ }^{9}$ As fraturas vertebrais são as mais frequentes (49\%), constituindo fatores preditivos para outras fraturas e condições incapacitantes. ${ }^{9}$ Aos 80 anos, mulher da raça branca tem risco cumulativo anual de apresentar fratura de quadril aproximadamente de $3 \%$. A morbimortalidade das fraturas por osteoporose aumenta exponencialmente com a 
idade, sendo que sua frequência estará duplicada nos próximos 25 anos, em consequência do envelhecimento da população. ${ }^{9}$

O envelhecimento é fase na qual as doenças interagem com as perdas funcionais na maioria dos indivíduos. A melhora do estado funcional dos indivíduos idosos diminui mortalidade, internação hospitalar e institucionalização. ${ }^{11}$ As atividades instrumentais da vida diária (AIVD ou IADLs), avaliadas através do questionário de Lawton, ${ }^{12}$ determinam se o indivíduo pode viver sozinho, utilizando recursos disponíveis no meio ambiente, refletindo, assim, a capacidade do idoso adaptar-se ao seu meio. ${ }^{13} \mathrm{Na}$ velhice, a manutenção da independência, assim como da autonomia, estão intimamente ligadas à qualidade de vida.

O estudo multicêntrico do European Vertebral Osteoporosis Study Group (EVOS) pesquisa os fatores de risco para osteoporose, avaliando os dados demográficos e antropométricos, história familiar de fratura de fêmur após os 50 anos de idade, história reprodutiva e menstrual, uso de medicações contendo cálcio, ingestão de alimentos ricos em cálcio, antecedentes pessoais, impacto da doença sobre a saúde geral do indivíduo (determinação do estado funcional), atividade física, tabagismo e alcoolismo. ${ }^{14}$

Este trabalho foi realizado em mulheres com idade igual ou superior a 60 anos, com o objetivo de predizer a chance da doença osteoporose através do questionário EVOS, relatar a percepção da doença através da avaliação do questionário de AIVD e comparar o perfil dos fatores de risco e o autocuidado pelo AIVD nas mulheres com osteoporose.

\section{METODOLOGIA}

Foram selecionadas mulheres na pósmenopausa, com idade igual ou superior a 60 anos, que participavam da Universidade Aberta da Terceira Idade (UNATI) na Universidade Católica de Brasília (UCB). Inicialmente, as mesmas preencheram ficha de identificação com os dados sociodemográficos e o questionário EVOS. ${ }^{14} \mathrm{Em}$ seguida, aplicou-se o questionário de Atividades Instrumentais da Vida Diária (AIVDs) de Lawton, ${ }^{12}$ e realizou-se o exame de densitometria óssea nas incidências de coluna lombar e fêmur, com o equipamento Lunar-GE, para diagnóstico da osteoporose conforme critérios da OMS. ${ }^{15}$

Este estudo foi aprovado pelo Comitê de Ética em Pesquisa da Universidade Católica de Brasília (CEP-UCB), parecer $n^{\circ}$ 017/2005. Todos os participantes foram previamente informados sobre o objetivo da pesquisa e procedimentos realizados e assinaram o termo de consentimento livre e esclarecido.

Após análise dos dados dos questionários EVOS e de AIVD, realizou-se o ajustamento de uma regressão logística, considerando-se como variável dependente a osteoporose $(\operatorname{sim}=1$ e não $=0)$ e um conjunto de variáveis independentes. Para o questionário EVOS, procedeu-se a uma análise bivariada entre a variável dependente e todas as independentes. Foram consideradas significativas aquelas com o p-valor menor que 0,15. Em seguida, realizou-se análise multivariada com as variáveis selecionadas no processo bivariado, utilizando-se um nível de significância de 5\%.

No questionário de AIVD a variável dependente, osteoporose, foi analisada com as variáveis independentes binárias "uso do telefone", "ir a locais distantes", "fazer compras", "preparar as refeições”, "arrumar a casa”, "fazer trabalhos manuais", "lavar e passar", "conseguir tomar os remédios" e "cuidar das finanças", sendo todas elas codificadas como $1=$ com ajuda e $0=$ sem ajuda. Procedeu-se à análise bivariada entre a variável dependente e as variáveis independentes, com a finalidade de verificar, entre estas últimas, quais seriam incluídas no modelo multivariado. Nesta fase, consideraram-se significativas aquelas nas quais o valor de $\mathrm{p}$ fosse menor do que 0,15. A seguir, procedeu-se à análise multivariada considerando somente aquelas variáveis selecionadas no processo bivariado. Nesta fase, utilizou-se nível de significância de $5 \%$.

Para se comparar as pacientes com e sem osteoporose com as AIVDs codificadas ( $1=$ não 
consegue, 2 =com ajuda parcial e 3 = sem ajuda), foi utilizado o teste estatístico não-paramétrico exato deMann-Whitney, mantendo-se como hipótese nula que os escores médios não diferiam entre os grupos. Utilizou-se nível de significância de 5\%.

$\mathrm{Na}$ análise dos dados, foi utilizado o programa de estatística SAS, versão 8.2.

\section{RESULTADOS}

Das 58 mulheres avaliadas através do questionário EVOS, com idade média de 67,3 \pm 4,2 anos, 16 (28\%) apresentavam osteoporose e 42 (72\%) não tinham osteoporose. Das 16 com osteoporose, três tinham idade entre 60-64 anos, 10 entre 65-69 anos e três acima dos 69 anos (figura 1).

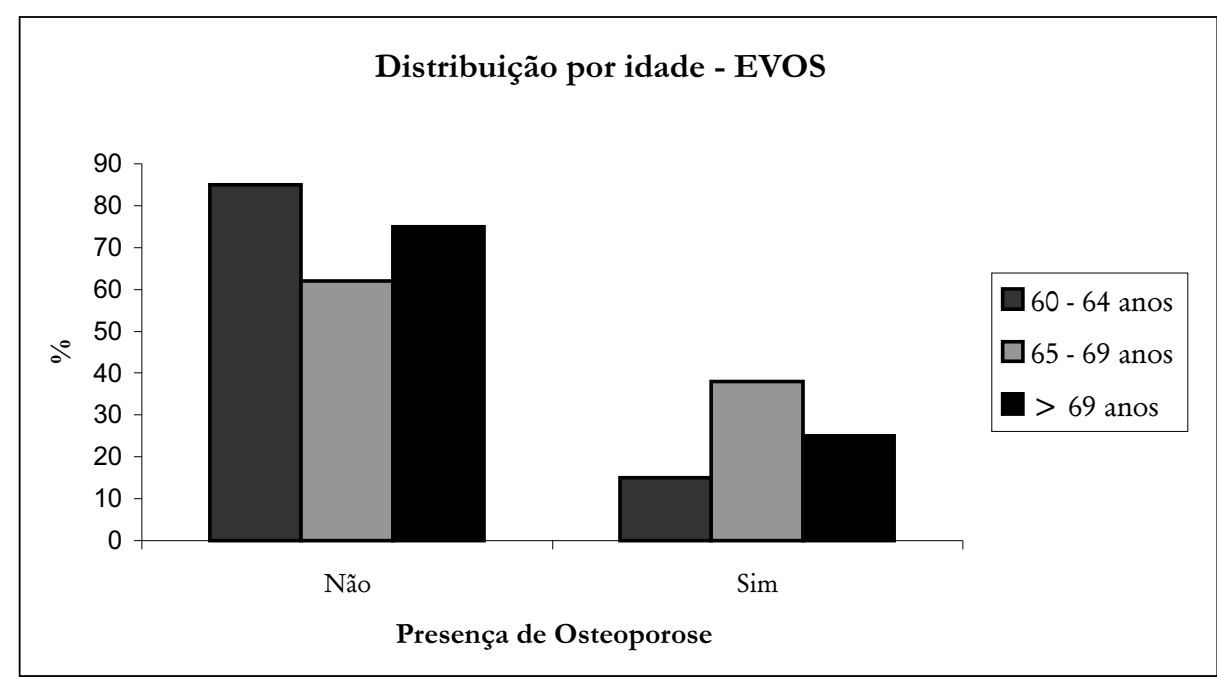

Figura 1 - Distribuição Etária das 58 Mulheres Avaliadas com Osteoporose.

$\mathrm{Na}$ análise bivariada, entre a variável dependente e as variáveis independentes ressaltadas no questionário do EVOS, foram selecionadas para a análise multivariada somente as seguintes variáveis: IMC $(\mathrm{p}=0,0332)$, nível de atividade física para as idades até 25 anos $(\mathrm{p}=0,1066)$ e uso de vitamina $\mathrm{D}(\mathrm{p}=0,1102)$ (tabela 1). 
Tabela 1 - Análise Bivariada entre Variável Dependente e as Independentes Ressaltadas do Questionário do EVOS. Brasília, DF, 2009.

\begin{tabular}{lrrrr}
\hline & Estimativa & $\begin{array}{c}\text { Erro } \\
\text { Padrão }\end{array}$ & $\begin{array}{r}\text { Estatística de } \\
\text { Qui-Quadrado }\end{array}$ & Valor p \\
\hline Idade & 0,0528 & 0,0615 & 0,7377 & 0,3904 \\
IMC & $-0,1930$ & 0,0906 & 4,5330 & 0,0332 \\
Idade Menarca & 0,0330 & 0,1590 & 0,0431 & 0,8356 \\
Idade Menopausa & $-0,0568$ & 0,0512 & 1,2296 & 0,2675 \\
Retirada de Útero & 0,2973 & 0,7781 & 0,1460 & 0,7024 \\
Retirada de ovários & $-0,4052$ & 0,8599 & 0,2221 & 0,6375 \\
Reposição Hormonal & $-0,3426$ & 0,6404 & 0,2862 & 0,5926 \\
Passado de Amamentação & 0,4563 & 1,1587 & 0,1550 & 0,6938 \\
Presença de Fratura & 0,0939 & 0,6393 & 0,0216 & 0,8832 \\
Frequência de consumo de álcool & $-0,2397$ & 0,3464 & 0,4789 & 0,4889 \\
Imobilização > 2 meses & $-0,3653$ & 0,8624 & 0,1795 & 0,6718 \\
Fumante & 0,4055 & 0,6191 & 0,4290 & 0,5125 \\
Atividade Física até os 25 anos & 0,4693 & 0,2908 & 2,6042 & 0,1066 \\
Atividade Física entre 25 e 50 anos & 0,3128 & 0,2898 & 1,1648 & 0,2805 \\
Atividade Física após 50 anos & 0,0816 & 0,3157 & 0,0668 & 0,7961 \\
Uso de Corticosteróides $>$ meses & 1,0113 & 1,0819 & 0,8738 & 0,3499 \\
Uso de Hormônio feminino & 0,1335 & 0,6528 & 0,0418 & 0,8379 \\
Uso de Cálcio & 0,6574 & 0,7595 & 0,7492 & 0,3867 \\
Uso de Vitamina D & 1,2528 & 0,7843 & 2,5515 & 0,1102 \\
Consumo de Leite até 25 anos & $-0,0590$ & 0,2671 & 0,0487 & 0,8253 \\
Consumo de Leite entre 25 e 50 anos & 0,2523 & 0,3219 & 0,6141 & 0,4333 \\
Consumo de Leite após 50 anos & 0,2666 & 0,3832 & 0,4842 & 0,4865 \\
\hline
\end{tabular}

Efetuando-se a análise multivariada, constatouse que nenhuma das variáveis foi conjuntamente significativa no modelo de regressão logística.
Controlando-se o efeito da variável IMC, não se encontrou associação entre a ingestão de vitamina D e osteoporose $(p=0,1269)$ (tabela 2$)$.

Tabela 2 - Análise Multivariada entre Variável Dependente e as Independentes Resultantes da Análise Bivariada. Brasília, DF, 2009.

\begin{tabular}{|c|c|c|c|c|}
\hline Variável & Estimativa & $\begin{array}{l}\text { Erro } \\
\text { Padrão }\end{array}$ & $\begin{array}{l}\text { Qui-Quadrado de } \\
\text { Wald }\end{array}$ & Valor $\mathrm{p}$ \\
\hline Intercepto & 3,3998 & 2,7204 & 1,5618 & 0,2114 \\
\hline IMC & $-0,2082$ & 0,1002 & 4,3180 & 0,0377 \\
\hline Atividade física para idades até 25 anos & 0,5039 & 0,2938 & 2,9422 & 0,0863 \\
\hline Variável & Estimativa & $\begin{array}{l}\text { Erro } \\
\text { Padrão }\end{array}$ & $\begin{array}{l}\text { Qui-Quadrado de } \\
\text { Wald }\end{array}$ & Valor $\mathrm{p}$ \\
\hline Intercepto & 1,9183 & 2,8477 & 0,4538 & 0,5006 \\
\hline IMC & $-0,1340$ & 0,1025 & 1,7106 & 0,1909 \\
\hline Uso de Vitamina D & 1,2233 & 0,8014 & 2,302 & 0,1269 \\
\hline
\end{tabular}


A análise do efeito isolado do IMC mostrou que, para cada aumento de uma unidade neste índice, a probabilidade de a mulher idosa não ter osteoporose foi de 21,3 $\%$, com intervalo de $95 \%$ de confiança $(1,5 \%$ a 44,9\%) (Tabela 3 ).

Tabela 3 - Análise do Efeito Isolado do IMC como Fator de Risco para Osteoporose. Brasília, DF, 2009.

\begin{tabular}{lcccc}
\hline Variável & Estimativa & Erro Padrão & $\begin{array}{c}\text { Qui-Quadrado de } \\
\text { Wald }\end{array}$ & Valor p \\
\hline Intercepto & $-4,3463$ & 2,4560 & 3,1317 & 0,0768 \\
IMC & 0,1930 & 0,0906 & 4,5330 & 0,0332 \\
\hline
\end{tabular}

Comparando-se as mulheres com e sem osteoporose por atividade mediante pontuação proposta no questionário de AIVD, identificou-se que aquelas com osteoporose apresentaram escores médios nas AIVDs significativamente menores do que aquelas sem osteoporose $(\mathrm{p}=0,042)$.

A análise bivariada do questionário de AIVD mostrou que somente as variáveis compra $(\mathrm{p}=0,006)$ e finanças $(\mathrm{p}=0,025)$ foram selecionadas para a análise multivariada (tabela 4). Ao se controlar o efeito de uma das variáveis em conjunto pelo modelo de regressão logística, observou-se que não houve associação entre elas. Por não ter sido significativa essa análise, passou-se ao estudo de cada variável separadamente.

Tabela 4 - Análise Bivariada das Variáveis do Questionário de AIVDs. Brasília, DF, 2009.

\begin{tabular}{lcccc}
\hline Variável & Estimativa & Erro Padrão & $\begin{array}{c}\text { Qui-Quadrado de } \\
\text { Wald }\end{array}$ & Valor p \\
\hline Intercepto & $-1,49$ & 0,38 & 15,70 & $<0,0001$ \\
Compra & 1,53 & 0,80 & 3,66 & 0,0558 \\
Finanças & 1,76 & 1,27 & 1,92 & 0,1659 \\
\hline
\end{tabular}

$\mathrm{Na}$ análise bivariada, encontrou-se que as mulheres que não conseguiam fazer suas compras tinham 7,39 vezes maior chance de ter osteoporose do que aquelas que conseguiam fazer esta atividade $(p=0,0063)$. Quanto às mulheres que não conseguiam cuidar de suas finanças, estas tinham 13,67 vezes maior chance de ter osteoporose do que as que conseguiam cuidar desta atividade $(p=0,0176)$.

Após o teste estatístico não-paramétrico exato de Mann-Whitney, comparando as mulheres com e sem osteoporose por atividade mediante pontuação proposta no questionário de AIVD, o escore total entre os grupos acima identificou que aquelas com osteoporose apresentaram escores médios menores do que aquelas sem osteoporose $(\mathrm{p}=0,042)$.

Analisando-se separadamente cada item, concluímos que as mulheres com osteoporose apresentam escores médios significativamente menores que aquelas que não têm osteoporose, para os itens: consegue fazer compras $(p=0,006)$, consegue fazer trabalhos manuais $(p=0,018)$ e cuidar das finanças $(p=0,014)$. 


\section{DISCUSSÃO}

No presente trabalho, utilizou-se a densitometria óssea como método padrão-ouro para diagnóstico de osteoporose. A doença foi identificada em $28 \%$ das mulheres deste trabalho, destacando-se $38 \%$ nas faixas de idade entre 65-69 anos.

Estudos epidemiológicos têm identificado a osteoporose como doença de impacto para Saúde Pública, delineando vários fatores de risco. ${ }^{13}$

A identificação dos fatores de risco dessa doença mostrou-se presente tanto no questionário EVOS como nas atividades da vida diária dessas mulheres idosas. Os fatores de risco envolvidos foram percebidos como a osteoporose influência na qualidade de vida dessas mulheres idosas.

O IMC, um dos fatores de risco no questionário EVOS encontrado nas mulheres idosas com osteoporose, foi de $26,23 \mathrm{~kg} / \mathrm{m}^{2}$, mostrando que as mulheres deste trabalho apresentavam sobrepeso. ${ }^{16}$ Assim, o IMC é considerado aqui fator preditor para ausência de osteoporose. Com base no questionário de AIVDs, observamos que o déficit no desempenho das atividades "fazer compras", "fazer trabalhos manuais" e "cuidar das finanças"

\section{REFERÊNCIAS}

1. Instituto Brasileiro de Geografia e Estatística (IBGE). Projeção da população do Brasil por sexo e idade 1980-2050. Rio de Janeiro: IBGE; 2008.

2. Pinto Neto AM, Soares A, Urbanetz AA, et al. Consenso brasileiro de osteoporose 2002. Rev Bras Reumatol 2002; 42 (6): 343-53.

3. Halbe HW. Síndrome do climatério. In: Halbe HW, Fonseca AM. Tratado de Ginecologia. 3.ed. São Paulo: Roca; 2000.

4. Trench B, Santos CG. Menopausa ou menopausas? Saúde e Sociedade 2005; 14(1): 91100.

5. Machado LV. Estratégia de saúde para a mulher climatérica. In: Fernandes, CE. (ed.).

Menopausa: diagnóstico e tratamento. São Paulo: Segmento; 2003. está correlacionado com a presença de osteoporose.

Os trabalhos feitos em diversas populações com o EVOS mostram que o questionário EVOS é válido, tanto para se estabelecer os fatores de risco para a osteoporose quanto para delinear os potenciais riscos de fratura vertebrais e nãovertebrais nos mais variados perfis populacionais. ${ }^{17,18}$ Estudos também validam o questionário de AIVDs na identificação de prejuízos na autonomia, o qual leva muita das vezes à dependência funcional, que declina com a idade, levando transtorno para a vida social.

\section{CONCLUSÃO}

Na presente investigação, não se identificaram fatores de risco com significância estatística para osteoporose. Isto pode ter ocorrido devido ao pequeno número de mulheres idosas estudadas. $\mathrm{Na}$ amostra estudada por meio do questionário EVOS, o IMC foi o fator preditor de ausência de osteoporose. A análise com o questionário de AIVDs mostrou déficit, com significância estatística, no desempenho para as atividades "como fazer compras", "fazer trabalhos manuais" e "cuidar das finanças” nas mulheres com osteoporose.
6. Carvalho MACR, Santos E, Silva de Sá MF. Avaliação multidisciplinar da osteoporose. Reprod Clim 1996; 11: 184-87.

7. Kanis JA. Bone density measurements in osteoporosis. J Intern Med 1997; 241: 173-75.

8. Bandeira F, Macedo G, Caldas G, et al. Osteoporose. . Rio de Janeiro: Medsi; 2000.

9. Marques Neto JF. Visão do reumatologista sobre osteoporose. Visão multidisciplinar 2002; 3: 1-3.

10. Fernandes CE, Melo NR, Wehba S, et al. Osteoporose pós-menopáusica. In: Fernandes CE; Melo NR; Wehba S. Climatério feminino: Fisiopatologia, diagnóstico e tratamento. São Paulo: Lemos; 1999.

11. Costa EFA, Porto CC, Almeida JC, et al. Semiologia do Idoso. In: Porto CC. Semiologia Médica. 4.ed. Rio de Janeiro: Guanabara Koogan; 2001. 
12. Paschoal SMP. Autonomia e independência. In: Papaléo Netto M. Gerontologia. São Paulo: Atheneu; 1996.

13. Scheidt-Nave C, Ziegler R, Raspe H. Epidemiology of osteoporosis. Med Klin 1998; 93 (2): 7-11.

14. O'neill TW; Cooper C, Cannata JB, et al. Reproducibility of a questionnaire on risk factors for osteoporosis in a multicentre prevalence survey: the european vertebral osteoporosis study. Int. J. Epidemiol 1994; 23(3): 559-65.

15. World Health Organization Study Group. Assessment of fracture risk and its application to screening for postmenopausal osteoporosis. Geneva: WHO; 1994.

16. ABESO. Índice de Massa Corporal. Disponível em URL: < http://www.abeso.org.br > .

17. Meyer HE, Falch JA, O'neill T, et al. The European vertebral osteoporosis study group: height and body mass index in oslo, norway, compared to other regions of Europe. Bone 1995; 17(4): 347-50.

18. Lunt M, Felsenberg D, Adams J, et al. Population-based geographic variations in DXA bone density in Europe: The EVOS Study. Osteoporosis lnt 1997; 7(3): 175-89. 